\title{
LT-SCM-SNI method for solving definite solution problems of three-interval composite nonlinear partial differential equations and
} its application

\author{
Xiaoxu Dong ${ }^{1}$, Shun $\mathrm{Li}^{1}$, Hong Guo ${ }^{1}$, Wei $\mathrm{Li}^{1}$, Wenjing $\mathrm{Li}^{1}$, and Qun $\mathrm{Liu}^{1}$ \\ ${ }^{1}$ Xihua University
}

November 5, 2020

\begin{abstract}
The definite solution problems of three-interval composite nonlinear partial differential equations (PDE) under different conditions is studied in the paper. Then the definite solution problems are solved by Laplace transformation - similar constructing method - Stehfest numerical inversion equation (LT-SCM-SNI method). Firstly, the definite solution problems of three-interval composite nonlinear PDE is transformed into the boundary value problems of three-interval composite linear of ordinary differential equation (ODE) by linearization and Laplace transformation (LT). Secondly, the solution in Laplace space of the boundary value problems of three-interval composite linear of ODE is obtained through using the similar constructing method (SCM). The solution in real space of the definite solution problems of three-interval composite nonlinear PDE is obtained through using the Stehfest numerical inversion equation (SNI) and linearization equation. Finally, the nonlinear spherical seepage model of threeinterval composite reservoir under infinite outer boundary conditions is solved through using the LT-SCM-SNI method. The influence of various parameters on dimensionless bottom-hole transient pressure is studied. The example shows the simplicity and practicability of the method presented in the paper.
\end{abstract}

LT-SCM-SNI method for solving definite solution problems of three-interval composite nonlinear partial differential equations and its application11 Project supported by Research on the coupling model of fracturing flowback in shale gas reservoir development, Talent introduction project of Xihua University (Z202068).

Xiaoxu Dong1,22Corresponding author. Email address: dongxiaoxu1028@163.com (Xiaoxu Dong)., Shunchu $\mathrm{Li}^{1}$, Hong Guo ${ }^{1}$, Wei Li ${ }^{1}$, Wenjing $\mathrm{Li}^{1}$, Qun Liu ${ }^{1}$

${ }^{1}$ School of Science, Xihua University, Chengdu, 610039, China

dongxiaoxu1028@163.com; lishunchu@163.com; 13541638376@163.com; supinf2003@aliyun.com; lwj2986345062@163.com; liuqun6661211@163.com

Abstract:The definite solution problems of three-interval composite nonlinear partial differential equations (PDE) under different conditions is studied in the paper. Then the definite solution problems are solved by Laplace transformation - similar constructing method - Stehfest numerical inversion equation (LT-SCM-SNI method). Firstly, the definite solution problems of three-interval composite nonlinear PDE is transformed into the boundary value problems of three-interval composite linear of ordinary differential equation (ODE) by linearization and Laplace transformation (LT). Secondly, the solution in Laplace space of the boundary value problems of three-interval composite linear of ODE is obtained through using the similar constructing method (SCM). The solution in real space of the definite solution problems of three-interval composite nonlinear PDE is obtained through using the Stehfest numerical inversion equation (SNI) and linearization equation. Finally, the nonlinear spherical seepage model of three-interval composite reservoir under infinite outer boundary conditions is solved through using the LT-SCM-SNI method. The influence of various 
parameters on dimensionless bottom-hole transient pressure is studied. The example shows the simplicity and practicability of the method presented in the paper.

Keywords:Definite solution problems; Nonlinear partial differential equations; LT-SCM-SNI method; Three-interval composite reservoir; Sensibility analysis

\section{Introduction}

Nonlinear partial differential equations and their systems as models for describing real-world phenomena often appear in the field of physical sciences and engineering. In practical applications, the solutions to the partial differential equation that satisfies some additional conditions, such as initial conditions and/or boundary conditions are a common concern. Therefore, the study of solutions to definite solution problems of nonlinear partial differential equations plays an important role in the study of nonlinear physical phenomena. Domestic and foreign scholars have done a lot of research in this area.

Ford and Pal et al. ${ }^{[6]}$ constructed a reliable numerical scheme for solving two-sided space-fractional partial differential equations. The use of numerical schemes usually solves a slightly perturbed problem. Yang and Deng ${ }^{[19]}$ put forward Riccati-Bernoulli sub-ODE method to construct exact traveling wave solutions, solitary wave solutions and peaked wave solutions for nonlinear partial differential equations. Han and Wang et al. [7] studied the existence and asymptotic behavior of solution for a class of boundary value problems of nonlinear higher order elliptic equations with two parameters. Sahadevan and Prakash ${ }^{[13]}$ showed how to use invariant subspace method to derive the exact solution of a time fractional nonlinear partial differential equation. This reference helps to derive multiple exact solutions. Madenci and Dorduncu et al. ${ }^{[10]}$ obtained the numerical solutions of linear and nonlinear partial differential equations (PDEs) by using differential operators. The boundary conditions of model they studied are Dirichlet and Neumann-characteristic boundary conditions. Yang and Goodyer et al. [18] introduced a new software tool for solving efficient solutions of parabolic linear and nonlinear partial differential equations. It is suitable for two different sample problems, which illustrate the flexibility and robustness of the tool. Abraham-Shrauner ${ }^{[1]}$ used the Power Index Method to identify the possible exact analytical nonlinear solutions of any of two characteristics of hyperbolic functions or any of three characteristics of Jacobian elliptic functions of nonlinear partial differential equations, which are invariant under the independent variables translations. Benamou and Froese et al. ${ }^{[2]}$ applied the classical "direct" technique involving Lie symmetries of partial differential equations to the boundary value problems of generalized Burgers equations with time-dependent viscosity coefficient and solved the particular sub-cases. However, this method has its limitations. Polyanin and Zhurov ${ }^{[11]}$ have derived the nonlinear differential-difference equations of motion for viscous incompressible fluid with finite relaxation rate and given the corresponding exact solutions to these equations. The results obtained can be used to solve some hydrodynamic problems for the differential-difference model of viscous fluid. Lee and Manteuffel ${ }^{[9]}$ proposed a natural framework for combining a Newton linearization and a FOSLL discretization approach for nonlinear partial differential equations. Yang and Deng et al. ${ }^{[19]}$ proposed the Riccati-Bernoulli sub-ODE method to construct the exact traveling wave solutions, solitary wave solutions and spike wave solutions of nonlinear partial differential equations and given the Backlund transformation of the Riccati-Bernoulli equation. This method provides a powerful and simple mathematical tool for solving some nonlinear partial differential equations in mathematical physics.

The study of reservoir seepage model is an important application of boundary value problem of differential equation. In recent years, the seepage theory has been continuously improved, but the theoretical and applied research on nonlinear seepage problems is still in primary stage. The study of nonlinear seepage mechanics has received more and more attention and has become a new direction in the development of modern seepage mechanics. Poon ${ }^{[12]}$ proposed a composite reservoir model of rock with fractal characteristics. And numerical solutions of the dynamic pressure of formation pressure is obtained by using the Laplace numerical inversion. Tong et al. ${ }^{[15-16]}$ obtained the asymptotic solution of the seepage model by inverse Laplace transformation. They discussed the influence of quadratic pressure gradient on pressure and described the difference between linear and nonlinear solutions. Zhang and Jia et al. ${ }^{[20]}$ established the nonlinear percolation model for threezone composite reservoir. Using variable substitution and Laplace transform, the analytical solution of 
Laplace space under the infinite boundary was obtained. By Stehfest numerical inversion, the real space solution was also obtained. However, they only suggested that we can use the Gaussian iterative elimination method to solve the equation to obtain the Laplace space solution of the bottom hole pressure. But they did not give the concrete expression of the solution. Dong et al. ${ }^{[3-4]}$ obtained the SCM for solving the boundary value problem of composite second-order linear homogeneous ODE. Dong and Liu et al. ${ }^{[5]}$ established the nonlinear three-region composite fractal reservoir. Using variable substitution and Laplace transform, the analytical solution of Laplace space under three different boundary conditions was obtained. By Stehfest numerical inversion, the real space solution was also obtained.

The methods given in the above literature are not suitable for complicated mathematical models and seepage models. Based on the above research, this paper studies the definite solution problems of three-interval composite nonlinear PDE under Dirichlet and Neumann-characteristic boundary conditions. In section 2, we propose the definite solution problems of three-interval composite nonlinear PDE and solve definite solution problem by linearization equation, Laplace transformation, similar constructing method and Stehfest numerical inversion equation. In section 3, the nonlinear spherical seepage model of three-interval composite reservoir under three kinds of outer boundary conditions (infinite boundary, constant pressure boundary and closed boundary) is solved by using the method obtained. In order to compare the influence of nonlinear term on bottom-hole pressure, the linear spherical seepage model is also solved in this section. In section 4 , the influence of various parameters on dimensionless bottom-hole pressure is studied. The influence of nonlinear terms and various parameters on the bottom-hole pressure is given in the form of characteristic graphs and tables. The results show that the nonlinear term and various parameters have great influence on the bottom-hole pressure. The example shows the simplicity and practicability of the method obtained in this paper.

2 The solution of the definite solution problem of three-interval composite nonlinear PDE

In this section, we first propose the following definite solution problem of three-interval composite nonlinear PDE:

where, , , ,, , , , , , are constants, and , .

Next, we study the solution to the definite solution problem . In order to linearize the governing equation, the following variable substitutions are proposed:

Substituting the equation (13) into the solution problem (12), the definite solution problem of nonlinear PDF (12) is transformed into the following the definite solution problem of linear PDF:

The Laplace transform is applied to the above definite solution problem (14) of dimensionless PDE, which will be transformed into the following boundary value problem of ODE:

Where is Laplace space variable.

Combining the outer boundary conditions and of the boundary value problem (15), the boundary value problem (15) is transformed into the following boundary value problem:

where .

If and are two linearly independent solutions to second-order linear homogeneous differential equations. According to equations (2)-(5), the following functions of guide solutions are define by and :

According to the similar constructing method ${ }^{[17-18]}$ for solving the boundary value problem of ODE, the solutions for inner, middle and outer intervals of the boundary value problem (16) are expressed respectively 
as follow:

,

where is called the similar kernel function of outer interval. Its expression is as below:

is called the similar kernel function of middle interval. Its expression is as below:

is called the similar kernel function of inner interval. Its expression is as below:

Using the Stehfest numerical inversion equation ${ }^{[20]}$ to the equations (21)-(23), the solutions for three intervals of the definite solution problem (14) in Real space are

where

According to the equation (13), the solutions for three intervals of the definite solution problem (1) in Real space are

3 The application of IT-SCM-SNI method

\subsection{Nonlinear Seepage flow model of three-interval composite reservoir}

This paper studies the physical model of nonlinear Seepage flow model of three-interval composite reservoir as Figure 1.

To formulate the nonlinear seepage flow model, the main assumptions are as follows:

2) Single-phase micro compressible fluid obeys Darcy's law and isothermal curve;

3) Reservoir has equal thick, each direction is horizontal and has a same nature;

4) Neglecting the capillary single-phase horizontal flow without gravity effect;

5) There is no additional pressure drop at the interface of two seepage interval;

6) Considering wellbore storage effects (in the beginning of opening well, the fluid stored in the wellbore starts to flow, the oil in the formation does not flow);

7) Formation pressure is initial reservoir pressure before producing;

8) The production well will be put into production with a fixed output of .

The nonlinear spherical seepage equations under cylindrical coordinates of the three-interval composite reservoir are obtained through the mass conservation equation (continuity equation), the equation of motion, the equation of state for liquid and rock ${ }^{[21]}$ :

The basic equation of seepage in inner interval:

The basic equation of seepage in middle interval:

The basic equation of seepage in outer interval:

where is reservoir pressure in interval, ; is liquid isothermal compression factor of interval, ; is rock isothermal compression factor of interval, ; is rock porosity in interval, dimensionless; is liquid viscosity of interval, ; is 
reservoir permeability in interval, ; is effective wellbore radius, ; is reservoir radius of interval, ; is well-bore radius, .

The reservoir pressures of three intervals are equal at of nonlinear spherical seepage model of the threeinterval composite reservoir. So the initial condition is

Inner boundary condition:

where is formation volume factor, dimensionless; is well-bore storage factor, ; is bottom-hole transient pressure, .

Convergence conditions:

Infinite outer boundary conditions:

where is initial reservoir pressure.

\subsection{The solution of nonlinear Seepage flow model of three-interval composite reservoir}

In order to facilitate the research and description, reference yield is introduced and dimensionless variables are defined as follows:

,,,,,,,,.

Substituting above equations into nonlinear spherical seepage model of the three-interval composite reservoir, the definite solution problem of dimensionless PDE is obtained as below:

In order to linearize the seepage equation, the following variable substitutions are proposed:

Substituting equation into definite solution problem, The definite solution problem of nonlinear PDF is transformed into the boundary value problem of linear PDF as below:

The Laplace transform .is applied to the above definite solution problem (32) of dimensionless PDE, which will be transformed into the following boundary value problem of ODE:

and are two linearly independent solutions of differential equations. and are two linearly independent solutions of differential equations. and are two linearly independent solutions of differential equations. Here and are respectively the first and the second class of modified Bessel functions of order .

We define the function as below:

According to equations - (20), functions of guide solution are as follows:

According to equation, similar kernel functions of outer interval is as follows:

According to equation, similar kernel functions of middle interval is as follows:

According to equation, similar kernel functions of inner interval is as follows:

According to equations -, solutions of the dimensionless reservoir transient pressure of inner, middle and outer intervals in the Laplace space, and are :

Using the Stehfest numerical inversion equation to the equation, the solution of inner interval the definite solution problem in Real space is

where 
According to inner boundary condition of the definite solution problem, the expression of dimensionless bottom-hole transient pressure of the nonlinear model in Real space is:

4 Characteristics of the nonlinear seepage

\subsection{The transient pressure characteristic curve}

The dimensionless bottom-hole transient pressure dynamic response curves of and of the nonlinear model of three-interval composite reservoir under infinite outer boundary condition are drawn in Figure 2 by compiling the corresponding program. Characteristic curve of bottom-hole transient pressure and transient pressure derivative under infinite outer boundary condition of three-interval composite reservoir can be divided into six seepage stages.

Phase I is the pure wellbore storage phase. The fluid seepage is principally affected by wellbore storage. Wells open and produce. The effusion seepage in the wellbore. The crude oil in the formation is at rest. The double log curves bottom-hole transient pressure and transient pressure derivative show a unit slope. The linear model is the same as the nonlinear model, that is, the nonlinear seepage is the seepage of the crude oil in the underground porous medium. Phase II is the transition seepage phase. The derivative curves are influenced by the skin factor and wellbore storage. Drilling, completion and other construction work may cause formation plugging, so there is pollution in the near well interval of oil well. Phase III is the seepage phase of inner interval. It reflects the formation characteristics of the inner interval. When the inner interval reaches the spherical flow stage, the transient pressure derivative curves no longer follows the " 0.5 line" rule, but is located below the " 0.5 line" due to the non-linear effect (The linear model follows the "0.5 line" rule). Phase IV is the seepage phase of middle interval. It is a reflection of the formation characteristics of the middle interval. Compared with the inner zone, the transient pressure derivative curve of middle interval will transform a step. If the physical properties of the middle interval (permeability, porosity and rock compression coefficient, etc.) are worse than the inner interval, the step of the transient pressure derivative curve will rise. If the physical properties of the middle interval are better than the inner interval, the step of the transient pressure derivative curve of middle interval will decline. Phase $\mathrm{V}$ is the seepage phase of outer interval. It is a reflection of the formation characteristics of the outer interval. The change of the transient pressure derivative curve of outer interval is similar to the phase IV. Phase VI is the total system spherical seepage phase. The transient pressure derivative curve converges to a horizontal line. It is reflection of infinite outer boundary condition.

\subsection{Parameter sensibility analysis}

In this section, we will study the effects of,,,,, , and on dimensionless bottom-hole transient pressure and transient pressure derivative of nonlinear seepage flow model of three-interval composite reservoir under infinite outer boundary condition. Transient pressure and transient pressure derivative characteristic curves of nonlinear seepage flow model affected by,,,,, , and are shown in Figures 3-10.

Firstly, we consider the effect of the quadratic pressure gradient on dimensionless bottom-hole transient pressure and transient pressure derivative by keeping the other parameters constant. Transient pressure and transient pressure derivative characteristic curves of nonlinear seepage flow model of three-interval composite reservoir under infinite outer boundary condition affected by is shown in Figure 3.

Figure 3 exhibits the transient pressure characteristic curves of nonlinear spherical seepage under different values of quadratic pressure gradient by keeping the other parameters constant. It can be seen that the quadratic pressure gradient has obviously impacted on the phase $\mathrm{II}^{\sim} \mathrm{VI}$ of spherical seepage phase. Furthermore, the bottom-hole transient pressure and transient pressure derivative decrease with the increase of the quadratic pressure gradient .

Secondly, we consider the effect of the on dimensionless bottom-hole transient pressure and transient pressure derivative by keeping the other parameters constant. Transient pressure and transient pressure derivative characteristic curves of nonlinear seepage flow model of three-interval composite reservoir under infinite outer boundary condition affected by is shown in Figure 4. 
Figure 4 exhibits the transient pressure characteristic curves of nonlinear spherical seepage under different values of the dimensionless parameter group by keeping the other parameters constant. It can be seen that the dimensionless parameter group has obviously impacted on the phases II-III of spherical seepage phase. Furthermore, the bottom-hole transient pressure and transient pressure derivative increase with the increase of parameter group.

Thirdly, we consider the effect of the reservoir radius of inner interval on dimensionless bottom-hole transient pressure and transient pressure derivative by keeping the other parameters constant. Transient pressure and transient pressure derivative characteristic curves of nonlinear seepage flow model of three-interval composite reservoir under infinite outer boundary condition affected by is shown in Figure 5 .

Figure 5 exhibits the transient pressure characteristic curves of nonlinear spherical seepage under different values of dimensionless reservoir radius of inner interval by keeping the other parameters constant. It can be seen that the dimensionless reservoir radius of inner interval has obviously impacted on the phase III of spherical seepage phase. Furthermore, the bottom-hole transient pressure and transient pressure derivative decrease with the increase of reservoir radius of inner interval .

Fourthly, we consider the effect of the reservoir radius of middle interval on dimensionless bottom-hole transient pressure and transient pressure derivative by keeping the other parameters constant. Transient pressure and transient pressure derivative characteristic curves of nonlinear seepage flow model of threeinterval composite reservoir under infinite outer boundary condition affected by is shown in Figure 6 .

Figure 6 exhibits the transient pressure characteristic curves of nonlinear spherical seepage under different values of dimensionless reservoir radius of middle interval by keeping the other parameters constant. It can be seen that the dimensionless reservoir radius of middle interval has obviously impacted on the phase IV of spherical seepage phase. Furthermore, the bottom-hole transient pressure and transient pressure derivative decrease with the increase of reservoir radius of middle interval. And the hump appears later with the increase of reservoir radius of middle interval .

Fifthly, we consider the effect of dimensionless parameter on dimensionless bottom-hole transient pressure and transient pressure derivative by keeping the other parameters constant. Transient pressure and transient pressure derivative characteristic curves of nonlinear seepage flow model of three-interval composite reservoir under infinite outer boundary condition affected by is shown in Figure 7.

Figure 7 exhibits the transient pressure characteristic curves of nonlinear spherical seepage under different values of dimensionless parameter by keeping the other parameters constant. It can be seen that the dimensionless parameter has obviously impacted on the phase $\mathrm{II}^{\sim} \mathrm{VI}$ of spherical seepage phase. Furthermore, the bottom-hole transient pressure and transient pressure derivative decrease with the increase of the parameter

Sixthly, we consider the effect of dimensionless parameter on dimensionless bottom-hole transient pressure and transient pressure derivative by keeping the other parameters constant. Transient pressure and transient pressure derivative characteristic curves of nonlinear seepage flow model of three-interval composite reservoir under infinite outer boundary condition affected by is shown in Figure 8.

Figure 8 exhibits the transient pressure characteristic curves of nonlinear spherical seepage under different values of dimensionless parameter by keeping the other parameters constant. It can be seen that the dimensionless parameter has obviously impacted on the phase IV $\sim$ I of spherical seepage phase. Furthermore, the bottom-hole transient pressure and transient pressure derivative decrease with the increase of the parameter

Seventhly, we consider the effect of dimensionless parameter on dimensionless bottom-hole transient pressure and transient pressure derivative by keeping the other parameters constant. Transient pressure and transient pressure derivative characteristic curves of nonlinear seepage flow model of three-interval composite reservoir under infinite outer boundary condition affected by is shown in Figure 9. 
Figure 9 exhibits the transient pressure characteristic curves of nonlinear spherical seepage under different values of dimensionless parameter by keeping the other parameters constant. It can be seen that the dimensionless parameter has obviously impacted on the phase $\mathrm{II}^{\sim} \mathrm{VI}$ of spherical seepage phase. Furthermore, the bottom-hole transient pressure and transient pressure derivative decrease with the increase of the parameter on the phase $\mathrm{II}^{\sim} \mathrm{VI}$ of spherical seepage phase. The bottom-hole transient pressure and transient pressure derivative increase with the increase of the parameter on the phase VI of spherical seepage phase.. And the hump appears later with the increase of reservoir radius of middle interval .

Finally, we consider the effect of dimensionless parameter on dimensionless bottom-hole transient pressure and transient pressure derivative by keeping the other parameters constant. Transient pressure and transient pressure derivative characteristic curves of nonlinear seepage flow model of three-interval composite reservoir under infinite outer boundary condition affected by is shown in Figure 10.

Figure 10 exhibits the transient pressure characteristic curves of nonlinear spherical seepage under different values of dimensionless parameter by keeping the other parameters constant. It can be seen that the dimensionless parameter has obviously impacted on the phase IV of spherical seepage phase. Furthermore, the bottom-hole transient pressure and transient pressure derivative decrease with the increase of the parameter

\section{Conclusions}

The definite solution problems of three-interval composite nonlinear PDE is transformed into the boundary value problems of three-interval composite linear of ordinary differential equation (ODE) by linearization and Laplace transformation. Then the solution in real space of the boundary value problems of three-interval composite linear of ordinary differential equation (ODE) is obtained by using similar constructing method and the Stehfest numerical inversion equation. The example shows the simplicity and practicability of the method obtained for solving the class of definite solution problems.

The LI-SCM-SNI method is simple and effective for solving definite solution problems of three-interval composite nonlinear partial differential equations, nonlinear seepage flow model of three-interval composite reservoir, etc.

\section{References}

1. Abraham-Shrauner B. Exact solutions of nonlinear partial differential equations. Discrete and Continuous Dynamical Systems - Series S, 2017, 11(4), 577-582.

2. Benamou J.D.; Froese B.D.; Oberman A.M. Numerical solution of the optimal transportation problem via viscosity solutions for the Monge-Ampere equation. Journal of Computational Physics, 2012, $260(2), 107-126$.

3. Dong X.X.; Li S.C.; Gui D.D.; et al. A study on solving the boundary value problem of three-region composite second-order linear homogeneous ODE. Energy Education Science \& Technology, Part A: Energy Science and Research, 2014 Volume (issues) 32(6), 6035-6048.

4. Dong X.X.; Liu Z.B., Li S.C. Similar constructing method for solving nonhomogeneous mixed boundary value problem of $n$-interval composite ode and its application[J]. MATHEMATICAL METHODS IN THE APPLIED SCIENCES, 2019, 42(5):1702-1723.

5. Dong X.X.; Liu Z.B., Li S.C. Similar constructing method for solving nonlinear spherical seepage model with quadratic pressure gradient of three-region composite fractal reservoir[J]. COMPUTATIONAL \& APPLIED MATHEMATICS, 2019, 38(2).

6. Ford N.J.; Pal K.; Yan Y. An algorithm for the numerical solution of two-sided space-fractional partial differential equations. Computational Methods in Applied Mathematics, 2015, 15(4), 923-8.

7. Han X.; Wang W.; Jiaqi M.O.; et al. Solution of singularly perturbed boundary value problem for nonlinear higher order elliptic partial differential equations with two parameters. Advances in Mathematics, 2015.

8. Jia Y.L.; Nie R.S.; Chen K.; et al. The flow model considering the quadratic pressure gradient and the well test characteristic curves. Journal of Southwest Petroleum University, 2007, 29(5), 69-71. 
9. Lee E.; Manteuffel T.A. FOSLL for nonlinear partial differential equations. Siam Journal on Scientific Computing, 2015, 37(5), S503-S525.

10. Madenci E.; Dorduncu M.; Barut A.; et al. Numerical solution of linear and nonlinear partial differential equations using the peridynamic differential operator. Numerical Methods for Partial Differential Equations, 2017, 33.

11. Polyanin A.D.; Zhurov A.I. Exact solutions of non-linear differential-difference equations of a viscous fluid with finite relaxation time. International Journal of Non-Linear Mechanics, 2013, 57(4), 116-122.

12. Poon D. International Meeting of the PetSoc. of. CIMSPE9-534, 1995.

13. Sahadevan R.; Prakash P. Exact solution of certain time fractional nonlinear partial differential equations. Nonlinear Dynamics, 2016, 85(1), 1-15.

14. Stehfest H (1970) Remark on algorithm 368: Numerical inversion of Laplace transforms. Communications of the ACM, 13(10):624.

15. Tong D.K.; Xue L.L.; Lian P.Q. Research on the numerical simulation of triple-porosity singular permeability model. Chinese Journal of Computational Mechanics, 2009, 26(4), 500-504.

16. Wang M.Y.; Tong D.K. Flow analysis of fluid in low permeability reservoir with double porosity including effects of quadratic gradient term and moving boundary. Chinese Quarterly of Mechanics, 2007, 28(3), 448-454.

17. Wen D.S. Engineering Fluid Mechanics. Higher Education Press, 2010.

18. Yang F.W.; Goodyer C.E.; Hubbard M.E.; et al. An optimally efficient technique for the solution of systems of nonlinear parabolic partial differential equations. Advances in Engineering Software, 2017, 103.

19. Yang X.F.; Deng Z.C.; Wei Y. A Riccati-Bernoulli sub-ODE method for nonlinear partial differential equations and its application. Advances in Difference Equations, 2015, 2015(1), 117.

20. Zhang L.; Jia Y.L.; Zhang F.X.; Yang X.T.; Qin S.Y. Analysis of considering quadratic pressure gradient nonlinear percolation in 3-zone composite reservoir. Journal of Daqing Petroleum Institute, 2011,35(5), 54-59.

\section{Hosted file}

Figures.pdf available at https://authorea.com/users/373408/articles/491111-lt-scm-snimethod-for-solving-definite-solution-problems-of-three-interval-composite-nonlinearpartial-differential-equations-and-its-application 